\title{
Paolo Sorrentino, la cultura della serie TV e Young Pope
}

\author{
Srećko Jurišić, Paula Jurišić
}

There must be some kind of way outta here

Said the joker to the thief

There's too much confusion

I can't get no relief

Bob Dylan, All along the watchtower

John Wesley Harding, 1967

Lultima opera di Paolo Sorrentino, Il papa giovane (2016, HBO - Sky Atlantic), segna una svolta importante nella poetica filmica del regista e sceneggiatore napoletano. Rispetto alle pellicole precedenti la serialità offre nuove possibilità narrative al regista generando così un genere e un linguaggio nuovi nella cinematografia italiana. L’articolo si prefigge come obiettivo l'analisi dettagliata della serie nonché l'esame dell'evoluzione della poetica di Paolo Sorrentino considerando che il regista sta preparando un film in due volumi.

Parole chiave: Paolo Sorrentino, cinema italiano, serie TV, The Young Pope, poetica

Zadnje delo Paola Sorrentina, Il papa giovane (2016, HBO - Sky Atlantic), predstavlja pomembno prelomnico v filmski poetiki neapeljskega režiserja in scenarista. $V$ primerjavi s prejšnjimi filmi ponuja pripoved $v$ več nadaljevankah nove možnosti naracije režiserju, kar odpira nove perspektive v italijanskem filmskem žanru in jeziku. Članek želi natačno analizirati serijo ter razvoj filmske poetike Paola Sorrentina upoštevajoč pedpostavko, da režiser pripravlja film v dveh nadaljevanjih.

Ključne besede: Paolo Sorrentino, italijanski film, televizijska nadaljevanka, Ilpapa giovane, poetika

a vasta progettualità e la grande mole di lavoro che si celano dietro il progetto 'papale' di Paolo Sorrentino ne lasciano intravedere la complessità che non si esaurirà in una sola stagione. Il cineasta partenopeo, infatti, intende sviluppare il proprio progetto con un follow up che vada al di là delle 9 ore e 6 minuti della prima serie avvalendosi del modello delle pluripremiate serie tv dal successo internazionale quali True Detective e Fargo (la cui prima stagione è l'addattamento dell'omonimo film dei fratelli Coen del '96) che stanno, per molti versi, rivoluzionando il mondo narrativo del piccolo schermo già da qualche anno. La lavorazione sarebbe durata più di due anni come ha affermato a più riprese Sorrentino e il punto di partenza, almeno quello visivo, sarebbe da ricercare in un'immagine curiosa:

\section{Il Papa vestito di bianco sulla neve fa lo sla- lom gigante tra le croci: è la prima scena che ho immaginato pensando a The Young Pope, e non la vedrete nella serie. Bellissima, ma nel processo di scrittura non ha trovato po- sto. Checchè ne dicano i miei detrattori non metto in un film una bella inquadratura a tutti i costi. Questa l'ho sacrificata, però mi piace raccontarla. ${ }^{\mathrm{I}}$}

L'immagine di Sorrentino richiama quella del papa Giovanni Paolo II, ipermediatico e quindi agli antipodi rispetto a Lenny Belardo, fotografato sulla neve anche se qualche dimostrazione d'interesse per l'universo vaticano la

\footnotetext{
Paolo Sorrentino, "The Young Pope. Paolo Sorrentino «Mi intrigava l'idea di un papa non riconducibile agli altri", Ciak magazine, (2 I ottobre 2016), http://www.ciakmagazine.it/the-young-pope-paolo-sorrentino-ami-intrigava-lidea-di-un-papa-non-riconducibile-ad-altria/\#BzpbtYiEjc6ODtlv.99 (21 ottobre 2016).
} 
si può trovare anche nella prefazione al volume fotografico dedicato a La grande bellezza dove Sorrentino raccoglie alcune impressioni delle sue prime visite a Roma («L'osservazione del grande, misterioso universo gravitante attorno al Vaticano era un'altra delle mie passioni $\gg)^{2}$ che poi ribadisce in varie interviste ( $\ll$ Roma è una città dove ci sono le categorie, molto più che altrove, a seconda delle zone puoi esplorare mondi. $\grave{E}$ bellissimo camminare in Vaticano e vedere tutto il mondo ecclesiastico che gravita lì intorno. Un cumulo di persone delle quali spesso a me sfugge il senso ultimo di quello che fanno»). ${ }^{3}$

Nel 2013, nel perodo in cui il regista stava montando La grande bellezza, nei cassetti gli scivolò la storia di un giovane papa americano, bello, conservatore e pieno di contraddizioni anche se lui era preso da un'altra santità, quella di padre Pio, passione nata dalla lettura della biografia del mistico di Pietrelcina firmata da Sergio Luzzatto che gli era piaciuta. A quel punto, Lorenzo Mieli, il produttore prodigio della Wildside, gli propone di farne una serie, ma Sorrentino nicchia perché

in tv di padri Pii ce n'erano stati già due, e un terzo, per quanto diverso, non mi pareva il massimo. Gli ho prospettato invece questa storia sul Vaticano e mi sono messo a scribacchiarla, sicuro che, in un Paese come il nostro, sarebbe stata lettera morta. Invece Mieli mi ha preso sul serio, ha combattuto per realizzarla; è intervenuta massicciamente Sky, poi siamo andati da Hbo, che per la prima volta ha coprodotto una serie europea. È un pionierismo di cui siamo fieri: ha aperto la strada a progetti come la saga della Ferrante e il Limonov di Carrère. I più grandi producer di serie tv al mondo chiedono a noi

2 Paolo Sorrentino, La grande bellezza. Diario del film, Milano, Feltrinelli, 2013, p. 9. Alessandra Coppola, Paolo Sorrentino si confessa: Il mio non è un lavoro per persone intelligenti», in Corriere della Sera, 2 maggio 2011).

3 Alessandra Coppola, Paolo Sorrentino si confessa:«Il mio non è un lavoroperpersone intelligenti», in Corriere della Sera, 2 maggio $201 \mathrm{I}$. italiani se abbiamo qualcosa di buono e dicono: bene, facciamola insieme. ${ }^{4}$

Il lavoro che ha accompagnato il Papa giovane proseguirà, dunque, offrendo nuovi spunti di riflessione con altre stagioni, indipendenti l'una dall'altra mentre l'idea della serialità pare si stia estendendo anche ai film di Sorrentino (è da poco uscito nelle sale Loro, il film su Berlusconi, in due parti.

Le serie americane menzionate appena più su, viste da Sorrentino su suggerimento, nient'affatto peregrino, di Bernardo Bertolucci (anch'egli abile nello sviluppare lunghe narrazioni filmiche ad alto tasso di epicità (vedi Novecento (1976) con le sue 5 ore e 17 minuti di durata) hanno avuto un peso nella scelta televisiva di Sorrentino la cui spiccata propensione per la narratività di celluloide ad ampio respiro l'avevano già avvicinato al mondo delle serie tv in passato (era tra gli autori della Squadra), ma senza che si realizzasse nulla di concreto:

Ne La Giovinezza, nel monologo di Jane Fonda, si parla di televisione. E sembra un po' un modo per mettere le mani avanti: la tv, dice Fonda, non è sminuente, ma anzi forse è proprio il futuro.

$\mathrm{Al}$ di là di quello che dice Jane Fonda in Youth, perché quello è un personaggio e dice quello che deve dire in quella scena, la mia idea è molto semplice: il racconto televisivo, che ti consente di lavorare sulle durate, su cose altrimenti impensabili al cinema, dove le gabbie, i tempi sono molto più rigidi,

4 Paola Zanuttini, "Papale papale: intervista a Paolo Sorrentino su "The Young Pope". La Repubblica, 26 settembre 2016. http://www. repubblica.it/venerdi/interviste/2016/og/26/news/papale_papale_ intervista_a_paolo_sorrentino_su_the_young_pope_-148575057/ (21 ottobre 2016).

5 «on sono uno spettatore bulimico di serie. Bertolucci mi ha suggerito di vedere True Detective, e io ho obbedito. Ė un progetto favoloso, che stravolge il genere. Poi ho visto le due meravigliose serie di Fargo. La buona televisione offre questa folgorante opportunità di prolungare il rapporto con la forza delle immagini cinematografiche e, allo stesso tempo, utilizzare un'ampiezza che è propria del romanzo e che il cinema spesso è costretto a sacrificare». ( $\mathrm{Pa}$ olo Mereghetti, «La grande bellezza» integrale. Sorrentino: «Cosi diventa estenuante come il racconto della vita», in Corriere della Sera, 25 giugno 2016, http://www.corriere.it/spettacoli/16_giugno_26/ grande-bellezza-integrale-racconto-estenuante-vita-sorrentino- $7 \mathrm{fc}$ cre9a-3afb-11e6-ao19-9orbc4c9foro.shtml\#, 21 ottobre 2016). 
e si deve obbedire a durate standard, è un'opportunità meravigliosa. Un'opportunità che io ho inseguito non da poco, devo dire. $\mathrm{Ma}$ per tanto tempo. Ho cercato di girare una serie televisiva con una certa libertà, sia creativa che economica, anche tanti anni fa. Ma non c'erano i presupposti. C'era una specie di dittatura del funzionario televisivo. Che diceva si fa così, devi dire questo, c'è questo pubblico e c’è quest'altro. E questo smorzava tutti i miei entusiasmi. Poi, finalmente, il mercato italiano è cambiato. Finché non c'è stato un cambiamento, esatto. Un'accelerata che è stata impressa dagli americani, che hanno dato vita a delle serie dove si è intelligentemente preso tutto il buono del grande cinema e tutto il buono delle possibilità televisive, e anche tutto il buono del romanzo. Perché la serie televisiva ha un'ampiezza di racconto e una capacità di divagazione che ti puoi permettere nel romanzo.

Quindi anche lei crede che le serie tv siano la "movaletteratura"?

Le serie televisive, per me, sono come quei figli particolarmente belli che vengono fuorida due genitori mediamente belli. Insomma, una serie televisiva può veramente essere il figlio bellissimo della letteratura e del cinema. ${ }^{6}$

Sorrentino, in un'altra intervista, dichiara anche:

Da sensazione e che la dimensione seriale sia congeniale al suo stile, gli conceda piu respiro. In parte è vero. Mi consente di mettere in campo molto materiale e allo stesso tempo mi obbliga a concentrarmi sul piano narrativo, che al cinema non considero determinante, mentre è fondamentale per tenere sulla distanza di dieci puntate. Sono un grafomane, scrivo tantissimo e nello spazio dilatato della serie riesco ad appagare la mia voglia di di

6 Gianmaria Tamaro, Paolo Sorrentino lancia la serie tv 'The Young Pope': "Il mio Papa vuole una Chiesa piu islamica", in Huffington Post, in ottobre 2016, http://www.huffingtonpost.it/2016/10/11/the-young-pope-sorrentino-papa-vuole-chiesa-islamica_n_12437424.html, 21 ottobre 2016)

\section{gressione, di moltiplicazione delle piste e dei personaggi che di puntata in puntata posso- no evolvere da comprimari a protagonisti c viceversa.}

Essendo Sorrentino un regista particolarmente attento alla scrittura, la serialità gli offre delle possibilità inusitate, delle vie non praticabili, insomma con il formato standard dei film. E sono le potenzialità che egli sfrutta sia dal punto di vista della densità della scrittura, molto stratificata, che della sua trasposizione filmica. ${ }^{8}$ A nostro parere, Sorrentino viaggia verso una forma ibrida dal momento che alle regole della spezzettata serialità $\mathrm{tv}$ non si piega in maniera rigida $\mathrm{e}$ che le lunghezze espressive per ottenere determinate atmosfere sono state già uno dei punti forti (e deboli, secondo una parte della critica) dei suoi film (Youth e La grande bellezza, su tutti). Il progetto berlusconiano, appena uscito, come anche la seconda stagione del progetto dedicato ai papi, daranno delle delucidazioni in questo senso e fanno parte della risposta 'europea' alle serie americane che si sono sovrapposte, negli ultimi anni, al cinema concedendosi certi lussi nel racconto che la coazione alla 'frettolosità', imposta dalle major e dal codice della consuetudine, precludeva o che, d'altro canto, lo schema episodico delle serie aveva obliterato del tutto. Sorrentino

s.n., "The Young Pope". Paolo Sorrentino «Mi intrigaval' idea di un papa non riconducibile agli altri», cit.

$8 \quad$ Altri esempi in questo senso: «Vous vous êtes entouré d'autres scénaristes familiers de la télévision. Est-ce pour intégrer la mécanique d'écriture de la série? Pas spécialement. J'ai suivi les règles qu'impose la série télé comme par exemple les cliffhangers (rebondissement, ndlr) qui terminent les épisodes, mais j’ai vraiment profité de la liberté que la longueur me donnait pour essayer de faire ce qu'en tant que cinéaste je ne peux plus faire : c'est-à-dire un grand film d'auteur qui dure plusieurs heures. Quelle autre liberté y avez-vous tronvé ?J'ai eu plus de place pour créer des moments de synthèses. Dans un film, ce sont des séquences assez brèves, des scènes mémorables, intenses - parfois même une simple image - qui vont tout résumer et permettre d'imprimer le souvenir de ce film dans le temps. Les séries télé manquent souvent de ces moments : même lorsqu'elles sont belles, on les oublie. Avec The Young Pope, mon défi était d'essayer de créer ces moments intenses, surprenants, des sommets de l'histoire qu'il faut, en quelque sorte, fixer.» (Isabelle Poitte, Paolo Sorrentino, réalisateur de "The Young Pope": "J'avais besoin de la dualité de Jude Law", in Télérama, 24 ottobre 2016 (http://www.telerama.fr/series-tv/paolosorrentino-j-avais-besoin-de-la-dualite-de-jude-law-pour-incarner-piexiii, 149062.php, 2 1 ottobre 2016). 
parte dai nuovi modelli americani ma per adeguarli alla sua poetica, insomma.

Ora, la conseguenza di queste mutazioni e osmosi tra generi consimili (vi accenna lo stesso Sorrentino in un'intervista a France Cultu$r e^{9}$ è in questo caso l'accentuata stratificazione di significati della serie e la sua densità contenutistica; esse rendono difficoltosa un'analisi dalle proporzioni accettabili in questa sede. Se ne potrebbe tentare, però, una lettura sintetica approcciando, per esempio, Young Pope dall'interessante sigla d'apertura. Quest'ultima compare dalla terza puntata (le prime due puntate erano più lunghe e invece di sacrificare una scena, il regista ha preferito sacrificare la sequenza iniziale) e contiene alcune delle chiavi di lettura possibili rivelandosi come un punto di partenza interessantissimo per un'analisi concentrata del Papa.

2. Questa la sigla: Jude Law, nei panni del pontefice, fresco di conclave, Pio XIII (un nome che lascia pensare ad un pontificato fortemente conservatore), passeggia sorridente in quello che può essere un salone dei Musei Vaticani mentre sullo sfondo vediamo susseguirsi delle opere d'arte che segnano le tappe importanti della storia della Chiesa inclusi alcuni momenti controversi come quelli raffigurati nei quadri di François Dubois (Massacre de la Saint Barthelemy le 24 août 1572) che raffigura il massacro degli ugonotti da parte della fazione cattolica) o nell' icona presente nel Monastero Mégalo Metéoron, in Grecia, che raffigura il Concilio di Nicea, che sancisce l'inizio di cesaropapismo; nella carrellata della sigla il regista non dimentica nemmeno la recente storia d'Italia, contenente non pochi punti oscuri: di Gerard van Honthorst, detto anche "Gherardo delle Notti", pittore influenzato dai maestri italiani e, in particolare, da Caravaggio, Sorrentino inserisce Adorazione dei Pastori che rimase gravemente danneggiata nell'attentato di via dei Georgofili a Firenze, compiuto da Cosa Nostra nella notte tra il 26 e il 27 maggio

9 Si tratta dell'intervista rilasciata a Olivia Gesbert il 3 I ottobre 2016 e ora disponibile al seguente link: https://www.franceculture.fr/emissions/la-grande-table-ıere-partie/young-pope-rencontre-avec-paolo-sorrentino.
1993. ${ }^{\text {10 }}$ La notte tra il 27 e il 28 luglio 1993 avvenne anche la strage di via Palestro a Milano (cinque morti e tredici feriti) e qualche minuto dopo esplosero due autobombe davanti alle chiese di San Giovanni in Laterano e San Giorgio al Velabro a Roma, senza però fare vittime: il giorno successivo due lettere anonime inviate alle redazioni dei quotidiani Il Messaggero e Corriere della Sera minacciarono nuovi attentati: la Chiesa, dunque, viene vista come parte attiva nelle complesse vicende politiche dell'Italia contemporanea come dimostra anche la scena dell'incontro tra Lenny Belardo e il premier, tratto da un modello piuttosto renziano, interpretato da Stefano Accorsi, in cui il neoeletto Sommo pontefice mostra al politico l'effettiva lunghezza della longa manus del Vaticano.

Jude Law è accompagnato, nella stessa sigla, da due cose: la prima è una specie di stella cometa, «la sua stella» (Matteo 2,I-I2.16), che l'accompagna di quadro in quadro per trasformarsi, alla fine della carrellata, in un meteorite e abbattersi sul papa Giovanni Paolo II visto da Maurizio Cattelan, uno degli artisti italiani più controversi in assoluto, nella scultura / istallazione La nona ora; ${ }^{\text {II }}$ il secondo elemento che accompa-

Io L'attentato in questione rientra nelle oscure dinamiche della cosiddetta Trattativa Stato - Mafia come dimostrano le motivazioni dei giudici fiorentini, oltre 500 pagine, della sentenza per il boss Francesco Tagliavia, accusato delle bombe del 1993: «Lo Stato avviò una trattativa con Cosa nostra, una trattativa che indubbiamente ci fu e venne quantomeno inizialmente impostata su un do ut des' per interrompere la strategia stragista di Cosa nostra. Lo scrivono i giudici della Corte d'assise di Firenze che hanno depositato le motivazioni della sentenza di condanna all'ergastolo di Francesco Tagliavia per le stragi del '93 a Firenze, Roma e Milano. E 'l'iniziativa - scrivono - fu assunta da rappresentanti dello Stato e non dagli uomini di mafia» (s.n., Stragi: «La trattativa mafia-Stato ci fu» Forza Italia non fu mandante delle stragi, in Il corriere fiorentino, $17 \mathrm{mar}$ zo 20I2, http://corrierefiorentino.corriere.it/firenze/notizie/cronaca/2012/12-marzo-2012/trattativa-mafia-stato-ci-fu-2003653215332. shtml?refresh_ce-cp, pagina visitata il 2 I ottobre 2016 .

I I La scultura s'intitola la Nona ora (1999, poliestere, resina, roccia vulcanica, tappeto, vetro, polvere metallica, lattice, cera, tessuto, con scarpe in cuoio e pastorale in argento) e raffigura, appunto, il papa Woytila schiacciato al suolo da un meteorite durante il suo incessante pellegrinare. Cattelan inizialmente aveva pensato il papa in piedi per poi segargli le gambe e inserire il meteorite. La Nona ora fa parte della cosiddetta Trilogia del Potere che racchiude, oltre alla la Nona ora, anche Him e Frank e Jamie. Il meteorite si abbatte dall'alto dei cieli sull' infaticabile pontefice riservandogli una fine piuttosto apocalittica considerata la fama mondiale di cui ha goduto durante il suo lunghissimo pontificato. Ciononostante, il livello di fama 
gna Jude Law nei panni di sornione flâneur nei titoli di testa è la canzone di Bob Dylan (qui nella versione di Devlin) All along the watchtower che gioca con quella specie di mistica laica, tanto cara al bardo di Duluth e ad altri cantautori della sua generazione con labili coordinate spazio temporali e riferimenti al libro di Isaia (2I, 5-9) che hanno per decenni confuso i critici nel tentativo di decrittarne i versi. La medesima apertura interpretativa ai limiti dell'arbitrarietà accompagna anche le azioni e le decisioni del pontefice sorrentininiano rendendolo assolutamente imprevedibile e, se si vuole, religiosamente inafferrabile. Alla stregua di una meteora il nuovo papa soppianterà il vecchio, così sovresposto nei media, inaugurando un pontificato all'insegna dell'assenza visto che Pio XIII si negherà ai fedeli.

Dice a proposito della sigla Sorrentino:

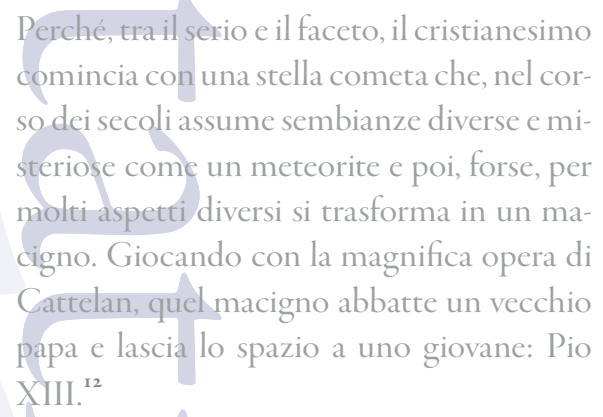

I richiami artistici sin dagli opening credits sono quindi tanti. La Nona ora di Cattelan con il suo sottotesto legato alle manifestazioni del potere (v. la nota 9 del presente lavoro) s' inserisce perfettamente nel disegno sorrentiniano legato

raggiunto, palesatosi attraverso tutti i paraphernalia del caso quali il merchandising ipertrofico e l'onnipresenza massmediatica generando non poche contraddizioni attorno a una figura devota alla Chiesa. L'istallazione controversa di Cattelan a Varsavia ha sollevato interrogativi circa l'ascesa vorticosa, da meteorite, del leader religioso cattolico e la sua fama. Non a caso il papa sorrentiniano eviterà di farsi vedere impedendo anche la produzione di gadget con la sua effigie. Su Cattelan si veda almeno Francesco Bonami, Nancy Spector (a cura di), Maurizio Cattelan, London Phaidon Press, 2003. Per l'interpretazione della Nona ora: Martin Kemp, Message frome the heavens, in Nature, n. 453, 2008, p. I 185.

I2 Lucio Perrotta, La sigla di The Young Pope spiegata da Paolo Sorrentino: "Quel Pio XIII somiglia a me", in Huffington Post, http://www.huffingtonpost.it/2016/11/18/quadri-sigla-young-pope_n_13074200.html, 21 ottobre 2016 alle stesse tematiche che pure sembra prevedere una trilogia:

Sorrentino, ha detto che il suo film migliore è "Il
divo" in cui raccontava il suo Andreotti. Adesso è
il turno del leader di Forza Italia.
«Sto scrivendo il film su Berlusconi, quando
lo farò lo interpreterà Toni Servillo».
Sembra affascinato dal potere, anche The young
Pope racconta cosa vuol dire muoversi nel potere
del Vaticano.
«Il tema del potere è dappertutto, è una del-
le tentazioni più forti del genere umano. Mi
viene da sorridere quando sento dire "Non
siamo governati bene": ci vuole saldezza mo-
rale per comandare. Che inevitabilmente si
erode, il potere prevede spesso l'abuso. È un
tema che mi affascina anche quando parlano
il carabiniere e il netturbino».

Inoltre, la passeggiata in solitario del papa nella sigla invita alla riflessione su un altro tema tanto caro a Sorrentino, quello della solitudine, strettamente legato al tema del potere. In una delle scene, il novello pontefice fa una sorta di elogio di chi rendendosi misterioso, negandosi ed isolandosi dal mondo o dal milieu in cui opera, accresce il proprio potere e la propria sfera d'influenza, esattamente come farà Pio XIII:

\section{Mi consenta, Santo Padre, questo che lei prospetta è un suicidio mediatico bello e buono.}

Come sono ingenue le università americane! Ma dico io, lei è francese, non le conveniva studiare lettere alla Sorbonna? Suicidio mediatico, dice lei? Adesso provi a seguirmi, se ne è capace.

\section{La seguo perfettamente.}

Chi è lo scrittore più importante degli ultimi venti anni? Faccia attenzione, non il più bravo, ma il più importante, quello che ha

13 Silvia, Fumarola, Paolo Sorrentino, dopo il Papa un film su Berlusconi: "Il potere, una delle tentazioni piu forti del genere umano", in Corriere della Sera, 8 aprile 2017 (http://www.repubblica.it/ spettacoli/cinema/2017/04/o8/news/paolo_sorrentino_berlusconi_ the_young_pope-162457314/,21 ottobre 2016). 
destato una curiosità così morbosa da diventare il più importante.

- Non saprei. Diciamo... Philip Roth.

No, è Salinger. Il regista cinematografico più importante?

Spielberg?

No. Kubrick. L'artista contemporaneo?

Jeff Koons o Marina Abramović.

No. Banksy. Il gruppo di musica elettronica?

- Non so nulla di musica elettronica.

E poi dice che Harvard è una buona università! Comunque, i Daft Punk. La più brava cantate italiana?

Mina.

- Brava. E lo sa qual è il filo rosso che unisce tutte queste figure che sono le più importanti nei loro rispettivi campi?

- No, non lo so.

- Non si fanno vedere. E non si fanno fotografare. $^{\mathrm{I} 4}$

L'interrogativo di Sorrentino, dalle tinte inevitabilmente autobiografiche, sembra ruotare attorno alla forza della solitudine ovvero dalla quantità di forza che un uomo solo (in questo caso al comando della Chiesa) può trarre dalla propria esperienza di orfano per farsi strada nella vita. La solitudine di Lenny va al pari passo con il suo potere, è isolato in Vaticano (persino Suor Mary e Dussolier, o anche il mentore Spencer gli voltano le spalle, a turno) e lo è anche sul piano personale a causa della sua condizione di orfano che, in ultima analisi, fa da propellente nel suo percorso di ricerca. In questo senso, la sigla d'apertura della serie si collega al finale in maniera circolare quando la cinepresa esce di scena uscendo di fatto dal mondo, passando attraverso le nuvole raffiguranti una figura crocifissa, una visione di Dio, si presume, dopo che i genitori gli hanno voltato ancora le spalle lasciandolo

14 Paolo Sorrentino, Il peso di Dio. Il Vangelo di Lenny Belardo, Torino, Einaudi, 2017, Pp. $24-25$ (d'ora in avanti PD seguito dal numero di pagina in coda alla citazione). solo nella solitudine definitiva. Anche dal puno di vista spaziale il percorso di Lenny si profila circolare: dalla macabra scena sognata in piazza San Marco, a Venezia, in cui da una pila di neonati morti ne emerge uno vivo si arriva, nell'ultimo episodio alla medesima piazza per l'explicit della serie. Su questo punto Sorrentino dice:

Nel primo episodio c’è una scena splendida in
piazza San Marco: da una piramide di neona-
ti apparentemente morti ne emerge uno vivo. Che
vuoldire?
«Non lo so, non lo so. A volte mi vengono
queste immagini, le trovo potenti, intense,
e decido di metterle, con disinvoltura, per-
ché nascono con un' intuizione istintiva. Poi
affiora un collegamento con la storia, una
plausibilità che, in questo caso, devo anco-
ra scoprire. Un bambino di sette anni abban-
donato ha necessariamente a che vedere con
la morte. E quella è una piramide di morte
perché quando i tuoi genitori ti abbandona-
no - non muoiono, ti abbandonano, ed è an-
cora più doloroso - non c’è niente di più vici-
no alla morte. Il fatto che Belardo, diventato
adulto, esca da questa morte e diventi una
guida dei vivi è una specie di resurrezione».

La resurrezione sognata da Lenny ha una sua valenza nell'economia del personaggio e della sua costante ambiguità, ma ne diremo più in là. Per ora basti ricordare che è un percorso inverso rispetto a quello compiuto da Cristo, che prima muore e poi resuscita. Ora, a non è dato saper se Lenny muoia o meno in seguito al malore perché, in fondo non è importante: ha compiuto la propria missione: nella folla radunatasi in Piazza San Marco ha trovato il proprio Dio credendo di individuare i propri genitori e vedendoli andarsene. Persino quest'ultimo fatto, per quanto dolente, perde in importanza nonostane essi siano stati l'oggetto di una vera e propria quête ${ }^{16} \mathrm{du}-$

is Paola Zanuttini, Papale papale: intervista a Paolo Sorrentino su "The Young Pope", cit.

I6 Per Sorrentino, non credente, Dio s'identifica sovente con qualche cosa che si desidera fortemente. Nel caso di Lenny si tratta dei genitori, un dettaglio, com' è noto che lo accomuna a Sorrentino stesso. In quel senso basti ricordare la scena di Youth (2015) in cui Fred 
rante l'intera serie impedendogli di governare la chiesa in maniera imparziale ed equilibrata perché Belardo guida la Chiesa finendo spesso ad essere guidato in maniera umana, troppo umana, dalle proprie paure e dalle proprie debolezze inducendo addirittura chi gli sta attorno a portare a suo cospetto dei genitori fasulli onde fargli credere che li abbia trovati. Il Giovane Papa va in crisi ad ogni acutizzarsi della sua condizione da figlio abbandonato, ma riesce, come conseguenza, a trovare sé stesso o ad andarci vicino nonostante le crisi d'identità (nelle puntate 7 e 8) che lo confondono, lo distraggono, gli fanno pensare di avere fallito.

Il giovane Pio XIII si accorge, continuando la sua ricerca che in un certo qual modo condivide con lo stesso regista, anch'egli orfano dall'età di diciasette anni, che in questo modo dichiara, adducendo il suo stesso esempio, che in fondo si è sempre ciò che si è come esseri umani mentre tutto il resto viene dopo. La nota frase, apocri$\mathrm{fa}$, di Novalis che Sorrentino mette in bocca ai suoi personaggi in Youth circa il ritorno alla casa paterna («Io sto sempre andando a casa, sempre alla casa di mio padre $\gg)^{17}$ vale anche qui e Lenny Belardo si affanna nella quasi disperata ricerca dei propri genitori che sembra che ogni suo gesto, inclusi i miracoli che gli vengono attribuiti siano gesti con cui o cerca l'approvazione da parte di chi lo ha abbandonato da bambino o cerca di attirare la loro attenzione con azioni fuori dal comune per colmare quell'assenza. Persino il suo negarsi ai fedeli può dare uno spunto in questa direzione:

\section{Il solo fatto che lei ha annunciato il suo primo viaggio pastorale e la prospettiva di una sua prima apparizione pubblica ha avu- to come riflesso un aumento del dodici per}

Ballinger (Michael Caine) e Mick Boyle (Harvey Keitel) contemplano la bellissima Miss Universo interpretata da Madalina Ghenea entrare in acqua e, consapevoli dell' irraggiungibilità della donna la chiamano Dio. Nella serie c'è una scena che conferma quanto appena detto nelle parole di Lenny: «Voi dovete rispondergli: 'Caro Jimmy, pensa a tutto quello che ti piace. Ecco, quello è Dio» (PD, 27)

17 Paolo Sorrentino, La giovinezza, Milano, Rizzoli, p. 129.

\section{cento delle richieste di partecipazione alla sua prossima messa in San Pietro. \\ La curiosità morbosa dell'assenza, Emi- nenza. So bene di cosa si tratta, essendo cre- sciuto come un orfano. (PD, 99)}

In generale, il pontificato di Belardo è un pontificato molto fisico, legato al corpo, nonostante egli paia una persona distaccata dalla fisicità. Il papa si allena (lo vediamo fare Pilates), e anche se all'apparenza rifiuta il buon mangiare quella nefasta diet cherry coke non è che un vezzo gastronomico come lo sono, per certi versi, anche le sigarette che il papa fuma costantemente. Il corpo, dunque, gioca un ruolo molto importante ed è declinato in varie sue espressioni: vecchiaia, obesità, giovinezza, bellezza, laidezza, spesso contrastanti in egual misura in cui lo è l'intero umanissimo pontificato di Lenny. A questo punto possono rientrare in gioco i riferimenti pittorici presenti nella sigla. Ci sono varie ipotesi circa le influenze pittoriche presenti nella serie con il solito Caravaggio che viene sovente associato all'arte di Sorrentino nonostante lo stesso regista si sia premurato a smentire tali influenze in qualche occasione. ${ }^{18}$ Il regista ne evidenzia, però, delle altre che per certi versi legano perfettamente all'opera di Cattelan mostrata in coda alla sigla. La Nona ora colpisce anche grazie all'estrema plasticità con cui è resa la dimensione fisica del papa polacco, talvolta cruda e iperrealistica anche se sempre contraddistinta da un certo pudore; è perfettamente in linea con la rappresentazione sorrentiniana della fisicità umana nella serie che deve molto, dice Sorrentino, a Lucian Freud e Otto Dix nei cui quadri non si fatica a rinvenire la medesima plasticità delle masse corporee con forme e rilievi forti. ${ }^{\text {I9 }}$ Inoltre, il

I8 «La luce caravaggesca dei quadri ricorre nella serie. Oltre ai lati oscuri di Lenny Belardo, c'è qualche altro personaggio che esce fuori dalle tenebre? Io trovo che si cominci ad abusare un po' troppo del termine "caravaggesco". Ogni qualvolta appaiono delle ombre o dei neri si tira in ballo Caravaggio. Ma vi assicuro che da quando lavoro col mio direttore della fotografia non abbiamo mai citato, neanche una volta, Caravaggio.» (Lucio Perrotta, La sigla di The Young Pope spiegata da Paolo Sorrentino, cit.).

19 «Lasciamo per un attimo da parte la sua passione musicale e parliamo di arte, altra fonte d'ispirazione. Le sue inquadrature hanno una bellezza pittorica: ci sono degli artisti che l' hanno particolarmente influenzata? 
concetto di quadro è stato chiamato in causa da Sorrentino stesso come una delle chiave di lettura della serie:

\section{Pensa che la serie piacerá ai cattolici? Si, se la guarderanno con il trasporto che merita un quadro. Quando osservi un dipinto non lo fai per controllare se corrisponda al catechi- smo o per capire se il pittore meriti di esse- re considerato un buon cattolico. Piacerà se staranno al gioco e si accorgeranno che parla alla loro umanità. ${ }^{20}$}

Da non trascurare, tra le influenze artistiche quella fotografica di Luigi Ghirri ${ }^{21}$ con i suoi paesaggi talvolta sospesi, che non sembrano realistici e sconfinano nel metafisico dechirichiano, spesso sono paesaggi privi di figure umane ma mai privi dell'intervento dell'uomo sul paesaggio, il tutto reso attraverso l'uso di colori delicati e non saturi, fondamentali sia nella poetica di Ghirri che in Sorrentino che se ne avvale per trasformare il Vaticano in un non - lieu freddo e assettico o in un'eterotropia foucaultiana. ${ }^{22}$

Abbiamo già fatto riferimento all'ambiguità tout court del papa sorrentiniano che sembra essere l'unica certezza, all'interno del paradosso chiave della serie: è prevedibile che il papa è imprevedibile. Torniamo un attimo alla presunta resurezzione menzionata da Sorrentino nella citazione poco più su. Chiaramente, essa ribalta lo schema biblico visto che rispetto al per-

Caravaggio, Lucian Freud e il tedesco Otto Dix. Soprattutto con quest ultimo credo di avere delle analogie nella composizione delle immagini» (Angelo Sica, Paolo Sorrentino: "Lui è meglio di me“, in Grazia, 19 ottobre 201 I, http://www.grazia.it/stile-di-vita/interviste/ paolo-sorrentino-lui-e-meglio-di-me, pagina consultata il 20 dicembre 2017)

20 Silvia, Fumarola, Paolo Sorrentino, dopo il Papa un film su Berlusconi: "Il potere, una delle tentazioni piu forti del genere umano", cit.

21 Altro nome che ricorre come influenza fotografica è indubbiamenti è Gianni Berengo Gardin ( $«$ I miei fotografi preferiti. Luigi Ghirri e Gianni Berengo Gardin», Paolo Sorrentino, Paolo Sorrentino. Passioni, piaceri, paure: confessione d'autore liberamente ispirata al questionario di Proust, in Vogue, i7 luglio 2017, http://www.vogue. it/news/vogue-arte/2017/07/17/paolo-sorrentino-intervista-vogue-italia-luglio-2017/, pagina consultata 20 novembre 2017. Vogue Italia, luglio 2017, n. 803 , pag. 42

22 Sulla prossimità dei due concetti Cfr. Marc Augé, Non-Lieux. Introduction à une anthropologie de la surmodernité, Paris, Seuil, 1992, p. 14I. Per Foucault cfr: Michel Foucault, Eterotopia, a cura di Salvo Vaccaro, Tiziana Villani, Pino Tripodi, Mimesis, Milano 2010 corso intrapreso da Gesù, quello di Lenny contempla prima la resurrezione e poi, nel finale, la morte. Né l'una né l'altra sono certe: la presunta resurrezione potremmo postularla come vera in quanto da orfano Lenny diviene papa, ma la scena macabra con i bimbi morti è soltanto sognata mentre della morte finale non siamo certi. L'incertezza dei due eventi fondamentali può far pensare, ad esempio, che il sacrificio di Cristo, quello raccontato nella Bibbia, non sia stato poi così grande; esso viene relativizzato, perchè, in fondo, egli è morto soltanto per pochi giorni per poi venir richiamato nei cieli. Nulla di particolarmente eretico o scandaloso: a Sorrentino «piace nelle storie belle [...] proprio il racconto della fatica di stare al mondo» dell'essere umano che, al livello materiale, non viene premiato con un'ascesa nei cieli, ma si fonde piuttosto in un tutt'uno con l'universo, al di là delle deità, come nell'ultima scena della serie. Si spiega così, dunque, l'inversione morte/resurezzione, con la semplice volontà di raccontare le contraddizioni dell'umano, questa volta nel contesto ecclesiasti$\mathrm{co}^{23} \ll$ Il clero è sempre stato rappresentato nella sua infallibilità o malvagità; noi lo raccontiamo per quello che è: fatto da esseri umani $\gg .{ }^{24}$ Dopo avergli concesso persino l'incontro con la meretrice (qui è una escort) pari a quello che ha Gesù, ${ }^{25}$ l'ambiguità di Lenny viene ri-

23 Nell'intervista a Paola Zanuttini il regista ribadisce che la razionalità dell'irrazionale e viceversa fanno parte dell'umano: «Vengo da una famiglia dove tutti i parenti avevano visto fantasmi o munacielli e raccontavano cose incredibili. Sono cose che mi hanno molto impressionato da bambino, influendo sul mio rapporto con la realtà e la paura. Ma oltre le superstizioni la materia è molto più affasçinante. Anche se uno come me fatica ad afferrarne il senso: l'altro giorno parlavo con una donna di una certa età che mi sembrava di un' intelligenza meravigliosa, che poi ha detto: "Ora vado a messa, come tutti i giorni". Mi ha colpito: la sua razionalità, la larghezza di vedute stridevano con la fede, c'è un corto circuito che non riusciamo a capire, come se credere nel trascendente fosse un attentato all'intelligenza. Invece la maggior parte degli studiosi del cattolicesimo sono fra gli intellettuali più attenti, profondi e speculativi, proprio perché sono abituati a porsi domande» (Paola Zanuttini, Papale papale: intervista a Paolo Sorrentino su "The Young Pope", cit.)

24 Silvia, Fumarola, Paolo Sorrentino, dopo il Papa un film su Berlusconi: "Il potere, una delle tentazioni piu forti del genere umano", cit.

25 «Una escort nella hall di un grande albergo vicino al Vaticano dove si sono rifugiati Lenny e Dussolier nella loro fuga notturna in cerca di sigarette, fotografando l'iride di Lenny: 'Che occhi! Questa è la prova dell'esistenza di Dio» $(\mathrm{PD}, 67)$. 
badita da molti altri dettagli oltre che dalle sue stesse parole: «Voiello e Lenny Belardo.- Sa una cosa, Santo Padre? Lei è bello come Gesù, ma non è Gesù. - Forse sono più bello, a dire il vero. Ma non lo dica a nessuno» (PD, 47). Poche pagine prima Suor Mary aveva detto a Lenny che in lui vedeva «il riflesso di Gesù» $(\mathrm{PD}, 3 \mathrm{I})$ mentre poi sentiamo Lenny definire come calunnia la propria santità ( $\mathrm{PD}, \mathrm{IO} 2)$ e leggiamo un dialogo come questo:

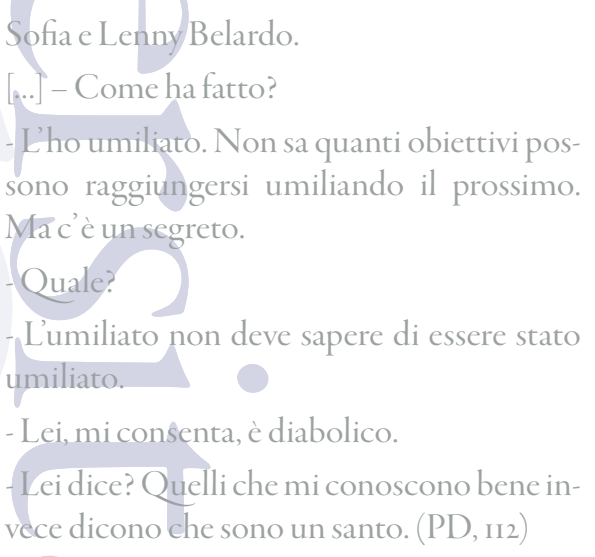

Lenny è qui direttamente associato a chi è stato il prediletto del Signore prima di Cristo stesso e chi si credeva più bello di Cristo stesso, al più luminoso degli angeli ovvero Lucifero e il gioco messo i piedi con il fine di umiliare il Premier italiano ricalca il gioco di Satana, carne di tanta letteratura. In poche parole il trucco dell'umiliare qualcuno senza darlo a vedere ricalca il massimo trucco del Diavolo di esistere convincendovi che non esiste come scrive Baudelaire nel Giocatore generoso; ${ }^{26}$ egli sarà anche bello come vediamo in Shakespeare ${ }^{27}$ o cercherà attraverso una «fede senza sorriso» di ritrovare suo padre tornando da dov'è venuto come scrive Eco nel Nome della rosa ${ }^{28}$ quasi parafrasando la frase di Novalis già citata.

$26 \ll$ Mes chers frères, n'oubliez jamais quand vous entendrez vanter le progrès des lumières, que la plus belle ruse du diable est de vous persuader qu'il n'existe pas» (Charles Baudelaire, Le Joyeur généreux, in Le spleen de Paris, Paris, Gallimard, 2010, p. 97.

$27 \ll$ The devil hath power/ To assume a pleasing shape». William Shakespeare, Amleto, Atto II, scena 2, v. 628.

28 «l diavolo non è il principe della materia, il diavolo è l’arroganza dello spirito, la fede senza sorriso, la verità che non viene mai presa dal dubbio. Il diavolo è cupo perché sa dove va, e andando va sempre
Il lato diabolico di Lenny è decisamente affascinante e letterario, come spesso in Sorrentino, simile a quello del Satana miltoniano, rappresentato come l'oggetto di ammirazione della storia, da imitare e celebrare come un vero eroe che lotta duramente per vincere i suoi stessi dubbi e le sue stesse debolezze per portare a compimento il suo piano di corruzione della specie umana; il più interessante e avvincente dei personaggi del Paradiso perduto, principalmente per la sua complessità e la sua astuzia e che si ribella poprio quando viene ripudiato, convinto com'era che Dio l'avrebbe nominato suo erede; si ribella cioè quando diventa orfano. Egli, come il Diavolo, si nasconde negandosi, questo è il suo trucco per raggiungere i propri scopi. Del resto, a sancire l'ambiguità della figura papale, anche un vero papa, un altro Pio, quello IX, aveva paventato una trattativa Stato della Chiesa - Diavolo qualora le circostanze lo dovessero richiedere nel discorso agli allievi del collegio Mondragone, il I6 maggio 1929: «Quando si trattasse di salvare qualche anima, di impedire maggiori danni di anime, ci sentiremmo il coraggio di trattare col diavolo in persona». Quasi un equivalente religioso di quella tra Stato e mafia di cui abbiamo scritto all'inizio.

Chiaramente, questa linea esegetica non ha lo scopo di ricercare componenti 'sataniche' nel tessuto della serie quanto piuttosto sottolineare l'ampiezza della visione sorrentiniana e la sua complessità che mira proprio all'estrema umanità del meno o del più umano dei papi il cui unico scopo è quello di ritrovare chi lo ha abbandonato settenne.

3. Avviandoci alla conclusione possiamo dire che l'universo vaticano, complesso e pericolosamente sfumato esige un'ampia tela su cui dipingere $^{29}$ e pare quasi che Sorrentino dia vita a un'opera ibrida, sospesa a metà strada tra un film

da dove è venuto» (Umberto Eco, Il nome della rosa, Milano, Bompiani, 198I, p. 364).

29 Nella già citata intervista apparsa su Télérama Sorrentino dice: «Pourquoi une série etpas un film? J'ai eu besoin de raconter le Vatican, le clergé, cet univers qui a beaucoup d'aspects et pour lequel un film aurait été limité. Même la série est limitée: il y a tellement à dire et à explorer» (Isabelle Poitte, Paolo Sorrentino, réalisateur de "The Young Pope": "J'avais besoin de la dualité de Jude Law", cit.). 
di quasi dieci ore e una serie vera e propria. Ciò che viene fuori è una sorta di Recherche di celluloide (con la scorza di mandarino richiamata nell'ultimo episodio a far da madeleine). Del resto, il capolavoro proustiano è sempre presente in Sorrentino, La grande bellezza è repleta di riferimenti ad esso, mentre in Young Pope l'opera viene apertamente citata in una scena della serie. ${ }^{3 \circ}$ Lenny Belardo, il papa non ancora cinquantenne creato da Sorrentino, serve da grimaldello al regista e allo spettatore per potersi introdurre nelle viscere dello Stato della Chiesa, nelle stanze del potere papale andando al di là del solito scandalo, uno dei tanti che scuotono la Chiesa contemporanea dalle fondamenta. In alcuni episodi della serie, svariati temi caldi (la pedofilia scarsamente condannata e la condanna fin troppo severa dell'omosessualità ecc.) vengono toccati o almeno accennati, persino in relazione con lo stesso giovane pontefice (il tentativo di ricatto da parte del segretario di stato Voiello, ma mai questi temi risultano essere centrali. Lo sono in quanto strettamente interconnessi allo spettacolo d'arte varia dell' umanità che gravita attorno al Vaticano. Quella sorrentiniana è una visione fortemente umanistica del sacerdozio e della religione, e quindi antropocentrica. Lo è perchè in fondo il suo obiettivo ultimo è quello di analizzare l'uomo e la sua difficoltà dello stare al mondo nei contesti più disparati, qui nel contesto dello spleen del sacro, per richiamare un'altra volta Baudelaire. Basta passare in rassegna la filmografia del regista per rendersene conto. Vi si scandagliano le tribolazioni, le passioni, i dubbi e le debolezze di uomini e donne di un'Italia a cavaliere del nuovo millenio. Ne La grande bellez$z a$, in cui Sorrentino va a «risciacquare i panni» nel Tevere, assistiamo al peculiare voyage au bout de la nuit (il riferimento a Cèline e dello stesso Sorrentino, è il suo libro preferito) attraverso l'altrettanto peculiare catabasi di Jep Gambardella, il Bardamu viveur de' noantri che persegue un ideale di beltà liminale, in bilico tra il tutto e il

\footnotetext{
30 «Comunque, Santo Padre, capisco la sua riluttanza sull'enciclica. Il suo predecessore mi faceva molto ridere quando diceva che un'enciclica è come la Recherche di Proust. Tutti la citano, ma nessuno la legge...» $(\mathrm{PD}, \mathrm{I} 7)$
}

niente che è tanto il néant sartriano («une nouvelle composante du réel vient de nous apparaître : le non-être») quanto il rien flaubertiano; Youth, magistralmente interpretato da Michael Caine e Harvey Keitel (ottimamente supportati da attori di prim'ordine come Rachel Weisz e Paul Dano o Jane Fonda), indaga l'umanità vegliarda, sull'orlo dell'abisso del trapasso. La scelta in questo caso cade sul pontefice probabilmente perché rappresenta l'uomo, almeno teoricamente, più costretto. Al punto che l'episodio della fuga di Lenny sembra quasi un omaggio a Habemus papam di Moretti dedicato ad un papa che fug$\mathrm{ge}^{3 \mathrm{I}} \mathrm{da}$ limitazioni e regole sociali che sono proprie della sua vocazione. L'indagine sorrentiniana crea così un uomo mosso dalla forte tensione alla metafisica che è invece è molto fisica nel senso che ciò che si crede e si vuole veder realizzato lo si vuol vedere fisicamente reale. Dalle iniziali dichiarazioni di scetticismo circa la propria fe$\mathrm{de},{ }^{32}$ si passa quasi un'accettazione di una visione non univoca della fede che verrà poi vista come qualcosa di personale e irripetibile. «Dio è a Venezia», è la frase di Lenny Belardo che non fa altro che confermarlo in una delle sue omelie analoghe, in vaghezza, alla canzone di Dylan posta in epigrafe di questo lavoro.

\section{Povzetek}

Zadnje delo Paola Sorrentina, Il papa giovane (2016, $\mathrm{HBO}$ - Sky Atlantic) predstavlja pomembno prelomnico v filmski poetiki neapeljskega režiserja in scenarista. V primerjavi z njegovimi zgodnjimi filmi (čeprav so bila nekatera njegova zgodnja dela precej dolga in so vključevala daljš̌ naracije, kot npr. La grande bel-

31 «Habemus Papam raccontava un pontefice in fuga. È un ottimo film, da spettatore sono innamorato di Moretti, ho tirato un sospiro di sollievo perché Nanni si ferma un attimo prima. Il mio Papa invece non vede l'ora di entrare in scena» (Silvia, Fumarola, Paolo Sorrentino, dopo il Papa un film su Berlusconi: "Ilpotere, una delle tentazionipiuforti del genere umano", cit.).

32 «Anche questa è resa 'umana' da Sorrentino: Il suo Pio XIII rivela al confessore di non credere in Dio, ma poi aggiunge: «Scherzavo». Però non scherzava tanto. Il suo è un rapporto con Dio ma capita a una certa età che la persona con cui abbiamo un legame d'amore si affievolisca. Un sacerdote ha la vocazione forte da giovane, poi un grande legame e negli anni crea un nuovo rapporto con Dio. L'amore è amore a tutti i livelli, cambia e si evolve». (Silvia, Fumarola, Paolo Sorrentino, dopo il Papa un film su Berlusconi: "Il potere, una delle tentazioni piu forti del genere umano", cit.). 
lezza (2013) in La giovinezza) dimenzije televizijske serije ponujajo nove možnosti Sorrentinu, ki delno sprejme nekatere nove poetike tako, da usmeri celoten projekt o papežu v televizijsko nadaljevanko desetih ur in pri tem ustvari novo zvrst v italijanski kinematografiji. Članek podrobno analizira Sorrentinovo zadnje delo, obenem pa ga umešča v razvoj njegove kinematografske poetike upoštevajoč predpostavko, da Sorrentino načetuje film $\mathrm{v}$ dveh nadaljevanjih.

Ključne besede: Paolo Sorrentino, italijanski film, televizijska nadaljevanka, Il papa giovane, poetika

\section{Summary}

The very last work by Paolo Sorrentino, The Young Pope (2016, HBO - Sky Atlantic) marks an important turn in the cinematic poetics of the neapolitan director and screenwriter. Compared to his earlier films (even though some of his earlier work was lenghty and encompassed extended narrative volumes, as in The Great Beauty (2013) or Youth) the dimension of the TV show opens to new possibilities to Sorrentino who accepts serial poetics only partially by orienting the entire 'papal' projects towards a tv movie of ten hours creating almost a new genre and a new language in the italian cinema. This article aims at the detailed analysis of Sorrentinos last work as well as at the accurate study of the evolution of his cinematic poetics considering that Sorrentino is currently working on a two-volume movie.

Keywords: Paolo Sorrentino, italian cinema, TV shows, The Young Pope, poetics

\section{Bibliografia}

Augé, Marc. Non-Lieux. Introduction à une anthropologie de la surmodernité. Paris: Seuil, 1992.

Baudelaire, Charles. "Le Joyeur généreux“. In Le spleen de Paris, 95-1 I0. Paris: Gallimard, 2010.

Coppola, Alessandra. "Paolo Sorrentino si confessa: $\ll$ Il mio non è un lavoro per persone intelligenti»”. Corriere della Sera, 2 maggio $201 \mathrm{I}$.

Eco, Umberto. Il nome della rosa. Milano: Bompiani, 1981.

Fumarola, Silvia. "Paolo Sorrentino, dopo il Papa un film su Berlusconi: "Il potere, una delle tentazioni più forti del genere umano". Corriere della Sera, 8 aprile 2017 http://www.repubblica.it/spettacoli/ cinema/2017/04/08/news/paolo_ sorrentino_berlusconi_the_young_popeI62457314\%.

Kemp, Martin. "Message frome the heavens". Nature, n. 453 (2008): II 85.

Mereghetti, Paolo. “«La grande bellezza» integrale. Sorrentino: «Così diventa estenuante come il racconto della vita»". Corriere della Sera, 25 giugno 2016. http:// www.corriere.it/spettacoli/r6_giugno_26/ grande-bellezza-integrale-raccontoestenuante-vita-sorrentino-7fccrega-3afbIre6-aor9-9orbc4c9foro.shtml\#.

Perrotta, Lucio, "La sigla di The Young Pope spiegata da Paolo Sorrentino: "Quel Pio XIII somiglia a me". Huffington Post 18 novembre 2116. http://www. huffingtonpost.it/2016/11/18/quadri-siglayoung-pope_n_13074200.html.

Poitte, Isabelle. "Paolo Sorrentino, réalisateur de "The Young Pope": "J'avais besoin de la dualité de Jude Law”. Télérama, 24 ottobre 2016. http://www.telerama.fr/series-tv/ paolo-sorrentino-j-avais-besoin-de-ladualite-de-jude-law-pour-incarner-piexiii,I 49062.php.

Sica, Angelo. "Paolo Sorrentino: „Lui è meglio di me”. Grazia, 19 ottobre 201r. http:// www.grazia.it/stile-di-vita/interviste/ paolo-sorrentino-lui-e-meglio-di-me.

Sorrentino, Paolo. La grande bellezza. Diario del film. Milano: Feltrinelli, 2013.

Sorrentino, Paolo, “The Young Pope. Paolo Sorrentino «Mi intrigava l'idea di un papa non riconducibile agli altri”. Ciak magazine, 21 ottobre 2016. http:// www.ciakmagazine.it/the-youngpope-paolo-sorrentino-ami-intrigavalidea-di-un-papa-non-riconducibile-adaltria/\#BzpbtYiEjc6ODtIv.99.

Sorrentino, Paolo. La giovinezza. Milano: Rizzoli, 2016. 
Sorrentino, Paolo. Il peso di Dio. Il Vangelo di

Lenny Belardo. Torino: Einaudi, 2017.

Sorrentino, Paolo. "Paolo Sorrentino. Passioni, piaceri, paure: confessione d'autore liberamente ispirata al questionario di Proust". Vogue, 17 luglio 2017, http://www. vogue.it/news/vogue-arte/2017/07/17/ paolo-sorrentino-intervista-vogue-italialuglio-2017.

Tamaro, Gianmaria. "Paolo Sorrentino lancia la serie tv 'The Young Pope': "Il mio Papa vuole una Chiesa più islamica". Huffington Post, II ottobre 2016. http:// www.huffingtonpost.it/2016/10/II/theyoung-pope-sorrentino-papa-vuole-chiesaislamica_n_I2 437424.html.

Zanuttini, Paola. "Papale papale: intervista a Paolo Sorrentino su "The Young Pope”. La Repubblica, 26 settembre 2016. http://www.repubblica.it/venerdi/ interviste/2016/09/26/news/papale_ papale_intervista_a_paolo_sorrentino_ su_the_young_pope_-I $48575057 /$. 